\section{Digitizer software for the microcomputer}

\author{
KEVIN KILLEEN \\ U.S. Department of the Interior, National Park Service \\ Data Systems Division, Washington, D.C. 20240
}

and

\section{R. BRUCE HULL IV \\ Department of Forestry \\ School of Forestry and Wildlife Resources \\ Virginia Tech, Blacksburg, Virginia 24061}

System Requirements. The programs described in this paper were written to interface a Tandy-Radio Shack TRS-80 Model I microcomputer with a Houston Instruments HIPAD digitizer. The TRS- 80 must have 48K RAM, at least one disk drive, and an asynchronousbit-serial RS-232-C. The digitizer should be equipped with the single-button cursor and the interface cable. The baud rate may vary from 110 to 4,800 bps and can be set by the software. The system is designed to scale the data in inches. DXY, a machine language program that provides the software interface, was assembled using the EDTASM $^{1}$ assembler. DIGITIZ/BAS, a TRS disk BASIC-interpreted program that drives the system, runs under the NEWDOS ${ }^{2}$ operating system.

Program Applications. The system performs a variety of digitizer-related functions and allows the user to tailor the digitized data to suit his specific needs. The HIPAD digitizer is very flexible and can be used in a variety of situations. It has resolution capabilities of .005 in., a repetition rate of up to 100 coordinates/sec, a relocatable origin with no loss of origin upon lifting the cursor, a digitizing area of $11 \times 11 \mathrm{in}$., and the capability of digitizing through nonmetallic materials up to .125 in. in thickness. The program allows the computation of area, length, and perimeter of two-dimensional figures, such as drawings, photographed objects, and mapped features. Furthermore, digitized coordinates outlining such features can be saved on diskette files to serve as inputs to digital plotting programs or for other user-determined applications. In addition, the BASIC program's structure is modular for easy program modification: One module accesses the digitized coordinates, several modules perform the various computations, and a final module displays the graphics. The user may access any of these modules with BASIC code and tailor the program to suit his specific needs.

System Operation. Digitized data can be entered in one of three modes. In the "point" mode, a single pair of digitized coordinates is transmitted to the microcomputer each time a button is pressed on the digitizer's cursor. In the "stream" mode, the digitizer transmits coordinates continuously. Finally, in the "switch stream" mode, continuous transmittal of coordinates occurs only while the cursor button is depressed.

An accumulator option exists that allows the addition and/or subtraction of areas and line lengths from those previously entered. This is often a great time saver when the areas being measured overlap.

Two output files may be created: Digitized coordinates can be serially output to diskette files, and the area and length computational results may be output in a format compatible with SPS, a statistical processing system for the TRS-80 microcomputer (Buhyoff, Rauscher, Hull, \& Killeen, 1980).

The system is capable of producing low-resolution graphical images of the digitized coordinates on the computer's display. Users with higher resolution CRTs or digital plotters may wish to modify the BASIC program or manipulate filed coordinate data to take advantage of their more sophisticated display devices.

Availability. The digitizer software is available from R. Bruce Hull IV, Department of Forestry, Virginia Tech, Blacksburg, Virginia 24061. Any request should include a blank 5.25 -in. soft-sector floppy disk and $\$ 2$ for printing costs of program documentation, program listings, and user guide.

\section{REFERENCE}

Buhyoff, G. J., Rauschen, H. M., Hull, R., \& Killeen, K. Microcomputer-resident comprehensive statistical analysis. Behavior Research Methods \& Instrumentation, 1980, 12, 551553.

\section{NOTES}

1. EDTASM is an editor-assembler program marketed by Radio Shack.

2. NEWDOS is a trademark of Apparat, Inc., Denver, Colorado.

(Accepted for publication February 5, 1982.) 